\title{
A Tutorial on Particle Filters for Fault Detection and Diagnosis of Hybrid Systems
}

\author{
Pazilat Mahmut, Jikai Lin and Ling Fu \\ College of Electrical Engineering, Xinjiang University, Urumqi, China \\ jikailin_xju@139com
}

\begin{abstract}
Hybrid dynamic systems have recently been a hot research topic. As the complexity of such systems is increasing day-to-day and system sizes are continually expanding, it can cause significant financial loss and loss of life in the event of system collapses. Therefore, ensuring the reliability and security of systems is of major importance in societal and economic terms. The research of particle filtering has made tremendous advances in the last three decades and has been demonstrated to be a good way to estimate the states of systems. So the combination of particle filtering with hybrid systems can be a promising method in fault detection and diagnosis. In this work, particle filtering algorithms are introduced and the emphasis is in their usage in hybrid systems fault detection and diagnosis. The review reveals the strengths and weaknesses of these particle filtering methods, and some examples are given to illustrate the practical application in detection and diagnosis based on these methods. Finally, future research directions and the difficulties that remained to be resolved are examined. It is anticipated that this paper will help researchers in this field understand the methodology better and provide a new concept and method in hybrid systems fault detection and diagnosis.
\end{abstract}

Keywords: hybrid systems, particle filtering, fault detection and diagnosis

\section{Introduction}

With increasing system complexity, safety has been the prime importance for most industrial systems. When these complex systems collapse significant losses arise. For example, on April 26, 1986, the world's worst nuclear accident happened at the Chernobyl Nuclear Power Plant in the Soviet Union which caused a large number of nuclear material leakage and brought great disaster to the surrounding ecological environment. Ensuring the reliability and security of the system, therefore, is of great importance and significance to the society and the economy. Systems' fault detection and diagnosis (FDD) has opened up a new way to improve the reliability of complex systems. The term 'fault' is generally defined as any abnormal phenomenon which can result in unexpected behavior within the systems or the events leading to the deterioration of the whole system performance which are caused by parts of the components' failing. FDD is the process to detect, isolate, and identify faults in a system. Fault detection determines whether faults are present. Following fault detection, fault isolation determines the size and time-variant characteristics of the fault [1]. Fault diagnosis includes fault isolation and fault identification. The exact definitions are made by the International Federation of Automatic Control (IFAC) [2]. When a fault occurs, all the variables or parts of them in the systems will show different characteristics than in their normal state. These differences contain abundant fault information. It is the task of fault diagnosis to find the fault features and use them to detect and isolate the malfunction.

Hybrid systems refer to a class of complex systems that contain both discrete and continuous dynamic events and their interaction. The mutual effects between the discrete behavior and continuous dynamic make the systems more complex and greater challenges 
have been brought to the fault diagnosis research. One of the most acceptable classifications of fault diagnosis methods is that fault diagnosis methods can be fundamentally divided into analytical model-based methods, signal processing-based methods and knowledge-based methods, which is proposed by German Professor Frank [3]. When the systems' mathematical models of the controlled process can be established precisely, the analytical model-based methods can be the first choice for FDD. Neither the signal processing-based methods nor the knowledge-based methods need the models of the complicated systems. However, the signal processing-based methods are limited for fault isolation and identification purposes. The knowledge-based methods generally require one to study a lot of samples so the ability to recognize early faults is insufficient [4]. Now a new classification of fault diagnosis methods is quantitative analysis method which is based on the analytical model method and data driven method and qualitative analysis method which includes qualitative simulation, graph theory and expert system [5]. Nearly all of the methods mentioned above have been applied in FDD for hybrid systems. This is illustrated by the method-based hybrid automata, hybrid bond graph method and method-based global petri nets. Each of them presents advantages and disadvantages.

Filtering is an important theory in signal processing. It refers to removing certain frequency in the signals by filtration to inhibit or prevent the interference. The generalized definition can be any information processing. Fault detection filter (FDF) was originally proposed by Beard in 1971 and Jones in 1973. It was the first time that the concept of filtering had any connection with fault diagnosis. Though FDF was firstly designed for linear systems, the thought of it was more significant than the method itself. From that beginning, the filtering method has been widely used in FDD and the research is mostly focused on nonlinear systems and some achievements have been made. The Kalman filter $(\mathrm{KF})$ is usually employed for state estimation and output prediction. But it depends on the system being linear and the noise and disturbances being Gaussian. To solve the problems in nonlinear systems, the extended Kalman filter (EKF) was proposed. But when the system is strong nonlinear, the filtering precision and fault diagnosis effect are not ideal. Particle filter (PF) had been proposed in 1950s. However, it was not taken seriously owing to the limit of the information processing speed and some intrinsic imperfections. Thanks to the rapid development of computer technology and the particle degeneration of PF being solved to some extent by N.Gordon et al. in 1993 [6], particle filtering has attracted increasing worldwide scholastic interest. The PF solves the Bayesian recursive relations by means of Sequential Monte Carlo (SMC) methods. It has been demonstrated that PF has a lower false alarm rate and missing report rate than EKF in fault diagnosis for nonlinear systems. The methodology is a good way to handle nonlinear systems and it exhibits the mainstream trend and research upsurge in the current decade. More than a thousand papers about particle filtering have been published since 2000. Among them, fault diagnosis occupies a large proportion.

This paper intends to provide the readers a good understanding on how to apply particle filtering in FDD for hybrid systems. The recent theoretical results and applications along this direction are summarized. Because of the nature of the paper, more emphasis is placed on concepts, applications and deficiencies of methods. Please look up the references for technical details. The rest of the paper is organized as follows. In Section 2, particle filtering method is surveyed. In Section 3 and 4, the state estimation of hybrid systems and how to carry out FDD using PF are discussed. It is the key point of the paper. In Section 5, some application examples of FDD for hybrid systems are introduced. Section 6 contains a brief summary and perspectives. 


\section{Particle Filtering Method}

Definition 1. A state space model of the nonlinear stochastic discrete time-varing system can be defined as:

$$
\begin{aligned}
& x_{k}=f\left(x_{k-1}, u_{k-1}\right)+v_{k}, \\
& z_{k}=g\left(x_{k}, u_{k}\right)+w_{k} .
\end{aligned}
$$

where $x_{k} \in R^{n}$ denotes the state vector at sequent time instant $k$ with a known initial probability density function (PDF) $p\left(x_{0}\right), u_{k} \in R^{p}$ denotes the input (control) vector at time $k, z_{k} \in R^{q}$ denotes the output vector, $v_{k} \in R^{n}$ is the state noise and $w_{k} \in R^{q}$ is the observation noise. $f_{k}(\bullet)$ and $g_{k}(\bullet)$ are known nonlinear state function and observation function. Without loss of generality, $v_{k}, w_{k}, x_{k}$ are mutually independent and the PDF of $v_{k}$ and $w_{k}$ are known. Filtering resolves the problems of estimating the conditional mean $\hat{x}_{k / k}$ and variance $P_{k / k}$ of the state for the present moment by taking advantage of the known observation $Z_{k}=\left\{z_{1}, z_{2}, \cdots, z_{k}\right\}$. The faults we talk about here are the failures when the system parameter values change into new values reflected in a change in the state transition function $f_{k}(\bullet)$ and the measurement function $g_{k}(\bullet)$ at time $k$. Such fault can be detected by the state observer using PF.

Let $D_{k}=\left\{\left(z_{i}, u_{i}\right), i=1,2, \cdots, k\right\}$ denotes the available measurement information at the time $k$. $x_{0: k}=\left\{x_{0}, x_{1}, \cdots, x_{k}\right\}$ denotes the set of all states up to discrete time $k$. It is assumed that the system is Markovian (i.e., such that $p\left(x_{k} \mid x_{0: k-1}, z_{1: k-1}, u_{k-1}\right)=p\left(x_{k} \mid x_{k-1}, u_{k}\right)$ ). The objective is to recursively construct the posterior PDF $p\left(x_{k} \mid D_{k}\right)$ of the state according to the measured output $D_{k}$ and assuming conditional independence of the measurement sequence for the states. So the PF works in two stages: prediction and update. The process can be seen in Figure 2. The prediction stage uses the state-transition in (1) to predict the state PDF one step ahead which is called the prior PDF. The upstate stage uses the latest measurement to correct the prior PDF via the Bayes inference.

PF represents the PDF by $N$ random samples or particles $x_{k}^{i}$ with their associated weights $w_{k}^{i}$. The normalized form is $\sum_{i=1}^{N} w_{k}^{i}=1$. At time instant $k-1$, the prior PDF $p\left(x_{k-1} \mid D_{k-1}\right)$ is represented by $N$ particles $x_{k-1}^{i}$ and the corresponding weights $w_{k-1}^{i}$. To approximate the posterior PDF $p\left(x_{k} \mid D_{k-1}\right)$, new particles $x_{k}^{i}$ and weights $w_{k}^{i}$ are generated. Assuming particles $x_{k}^{i}$ are extracted from a proposal distribution function $q\left(x_{k} \mid D_{k}\right)$ and the proposal distribution function is determined by predicting the next state from the system model (as a response to a given set of inputs), then it is possible to calculate the weights as 


$$
\begin{aligned}
w_{k}^{(i)} & =\frac{p\left(x_{k}^{(i)} \mid D_{k}\right)}{p\left(x_{k}^{(i)} \mid z_{1: k-1}, u_{k-1}\right)} \\
& \propto \frac{p\left(z_{k} \mid x_{k}^{(i)}\right) p\left(x_{k}^{(i)} \mid z_{1: k-1}, u_{1: k}\right)}{p\left(x_{k}^{(i)} \mid z_{1: k-1}, u_{1: k}\right)} \\
& =p\left(z_{k} \mid x_{k}^{(i)}\right) .
\end{aligned}
$$

then the resulting posterior PDF $p\left(x_{k} \mid D_{k}\right)$ at time $k$ can be approximated as discrete weighted approximation [7]

$$
p\left(x_{k} \mid D_{k}\right) \approx \sum_{i=1}^{N} w_{k}^{(i)} \delta\left(x_{k}-x_{k}^{(i)}\right)
$$

where $\delta(\bullet)$ is the Dirac function and $w_{k}^{i}$ denotes the $i$-th $(i=1,2, \cdots, n)$ weight. The weights are nonnegative, sum up to unity and can be determined according to the importance sampling principle.

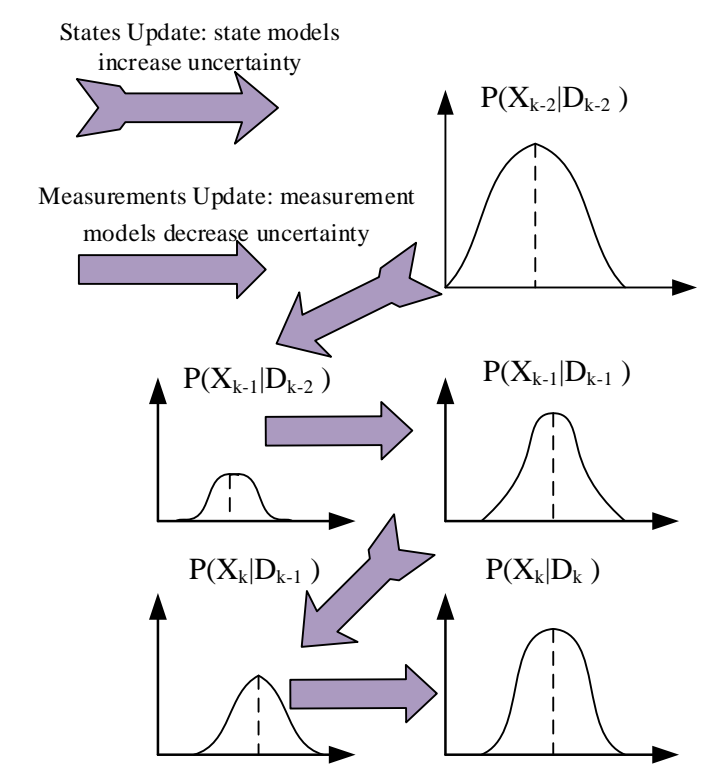

Figure 2. The General PF Framework

A common problem of PF is the particle degeneracy: after several iterations, the weights of all but one particle converge to zero. The serious disadvantage of it is that most of the computing time is wasted in propagating particles whose impact on the approximation of the posterior distribution is negligible. Therefore, particles must be resampled. The minimum number of particles which the PF needs to achieve the ideal approximation can be calculated by the effective sample size:

$$
N_{e f f}=\frac{1}{\sum_{i=1}^{N}\left(w_{k}^{i}\right)^{2}} .
$$

Thus, the degeneracy phenomenon can be detected by comparing the $N_{\text {eff }}$ with the set threshold value $N_{t h r} \in[1, N]$. If $N_{e f f}<N_{t h r}$, the resampling algorithm is just applied. The program of sequential importance resampling (SIR) is shown in Figure 3. 


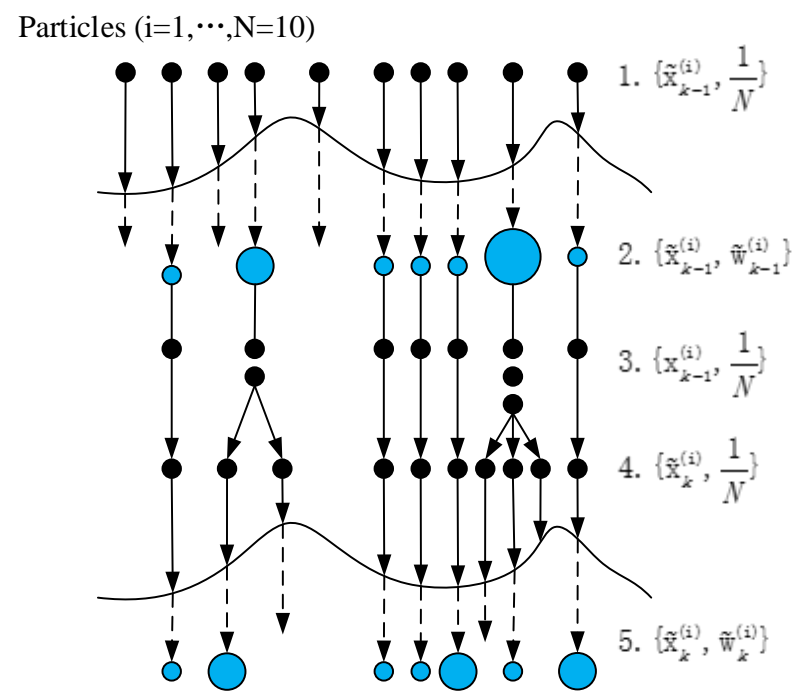

Figure 3. The Process of Sequential Importance Resampling

\section{Estimation of Hybrid System}

A hybrid system includes a set of discrete modes which represent operational models or fault states of the system and a set of continuous variables which model the continuous quantities that reflect the behavior of the system. Generally, a state is a mode plus a value for each continuous variable, while a mode is only the discrete part of the state [8]. There is no unified model for hybrid system and some well known models such as probabilistic hybrid automaton (PHA), jump Markov nonlinear system (JMNS) and hybrid bond graph (HBG) have been widely used in modeling for hybrid system. In order to facilitate discussion in this paper, the PHA model is chosen. To keep coherence and avoid confusion, the same symbols and meaning thereof have been applied hereafter.

Definition 2. A discrete-time PHA is described as a tuple $\langle X U Z T F\rangle$ :

- $\mathrm{X}$ denotes the hybrid state variables of the system which consists of $X=\left\{x_{d}\right\} \cup x_{c}$.

The discrete variable $x_{d}$ is the mode in the system and $x_{c}$ is the continuous variable state which captures the dynamic evolution of the system. It should be noted that $x_{d}$ is finite.

- The set of input variables $U=u_{d} \cup u_{c}$ of the system is composed of disjoint sets of discrete input variables $u_{d}$ and continuous input variables $u_{c}$.

- $\mathrm{Z}$ is the set of observable variables. $\left.P\left(Z_{k} \mid X_{k}\right), X_{k}=\left\{x_{d}\right\} \cup x_{c}\right)$ can be used for the distribution of observation in state $X$.

- $\mathrm{T}$ is the transition probability function that specifies $P\left(x_{d(k)} \mid x_{d(k-1)}, x_{c(k-1)}\right)$ or $P\left(x_{c(k)} \mid x_{d(k-1)}, x_{c(k-1)}\right)$ which is the conditional probability distribution over modes at time $\mathrm{k}$ given that the system is in state $\left(x_{d}, x_{c}\right)$ at time $k$-1. If it is independent of the continuous variables, then $P\left(x_{d(k)} \mid x_{d(k-1)}, x_{c(k-1)}\right)=P\left(x_{d(k)} \mid x_{d(k-1)}\right)$.

- $F$ denots the set of state function and $G$ is the set of observation function.

It is always assumed that the initial mode of the system is known or given as a distribution in the mode space. The same assumption applies to the initial state $x_{0} Z_{0}$ is insignificant because of the occurrence of observation at $k>0$. The transition functions from one mode to another are defined in a matrix $T$. 
The hybrid system state estimation problem can be formulated as follows. Firstly, like in the PF, calculate the PDF of the hybrid system: $p\left(X_{k} \mid Z_{1: k}\right)$. The distribution $p\left(X_{k} \mid Z_{1: k}\right)$ here is called belief state [9] which means that it gives the probability that $X$ is the true state of the system of each state $X$ at each time-step, given the observations so far. Secondly, track the belief state using the forward pass equation:

$$
\begin{aligned}
p\left(X_{k} \mid Z_{1: k}\right) & =\alpha p\left(Z_{k} \mid X_{k}\right) \int p\left(X_{k} \mid X_{k-1}\right) p\left(X_{k-1} \mid Z_{1: k-1}\right) d X_{k-1} \\
& =\alpha p\left(Z_{k} \mid x_{d}, x_{c}\right) \int p\left(x_{c(k)} \mid x_{d(k)}, x_{c(k-1)}\right) p\left(x_{d(k)} \mid x_{d(k-1)}, x_{c(k-1)}\right) p\left(X_{k-1} \mid Z_{1: k-1}\right) d X_{k-1}
\end{aligned}
$$

where $\alpha$ is a normalizing constant. A probabilistic graphical model [10] is given in Figure 4 (shown in [9]) to show the principle of state estimation for hybrid system.

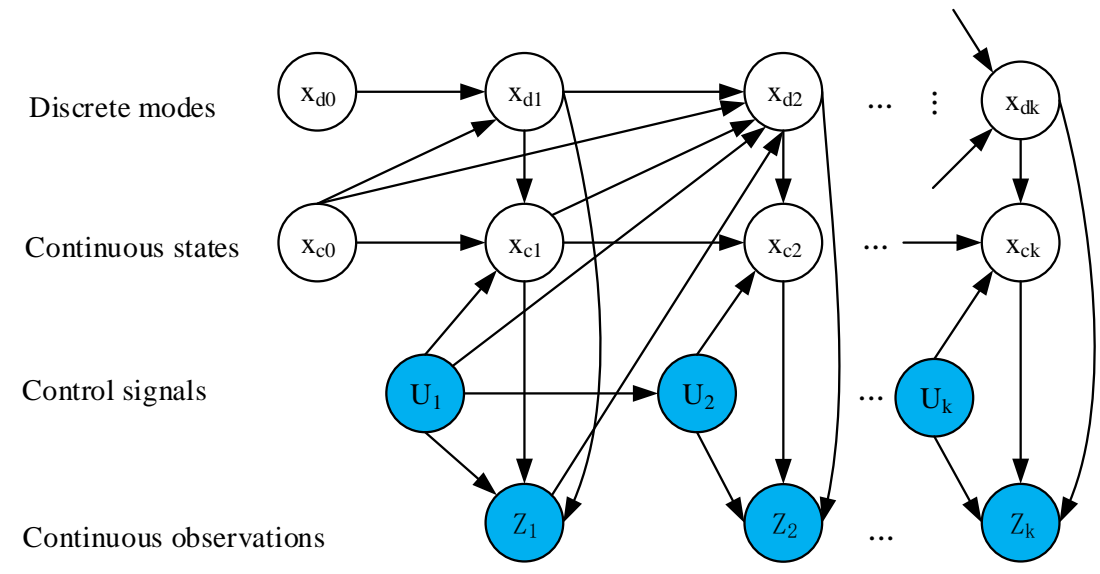

Figure 4. Model of Hybrid System State Estimation

With the help of the aforementioned preliminaries, the process of hybrid system state estimation using PF can be summarized as follows. Figure 5 (shown in [11]) illustrates the process vividly to help readers understand the program easier. Refer to [11] for details about the method.

Step 1: Initialization

- The mode at time $k=0$ is known as ${ }^{x_{d(0)}}$. Particle $X_{0}^{(i)}(i=1,2, \cdots, N)$ is given an initial distribution in mode ${ }^{x_{d(0)}}$ and then set $k=1$.

Step 2: Prediction

- For any mode $x_{d(k)}^{j}(j=1,2, \cdots, M, M$ is the number of modes. $)$ such that the transition probability function $T_{x_{d(k-1)} x_{d(k)}^{j}}$ from mode ${ }^{x_{d(k-1)}}$ to mode $x_{d(k)}^{j}$ is not zero, particle $\tilde{X}_{k}^{(i)} \sim p_{x_{d(k)}^{j}}\left(X_{k} \mid X_{k-1}^{(i)}\right)$.

- For each mode ${ }_{d(k)}^{j}$, calculate the importance weights $\tilde{w}_{k}^{(i)}=p_{x_{d(k)}^{j}}\left(Z_{k} \mid \tilde{X}_{k}^{(i)}\right)$. Step 3: Mode selection

- Get the average of the total particles weights for each mode ${ }^{x_{d(k)}^{j}}$ and multiply by the transition probability:

$$
\tilde{w}_{k}^{x_{d(k)}^{j}}=T_{x_{d(k-1)} x_{d(k)}^{j}} \sum_{i=1}^{N} \frac{\tilde{w}_{k}^{(i)}}{N} .
$$

- Track the mode: $x_{d(k)}=\arg \max \left(x_{d(k)}^{j}\right)$. 
- Normalize the weights of particles in mode ${ }^{x_{d(k)}}$.

Step 4: Resampling

- Resample $N$ new particles $X_{k}^{(i)}$ to replace the old ones $\tilde{X}_{k}^{(i)}$ in mode $x_{d(k)}$ according to the importance weights.

- Set $k=k+1$ and jump to step 2 .

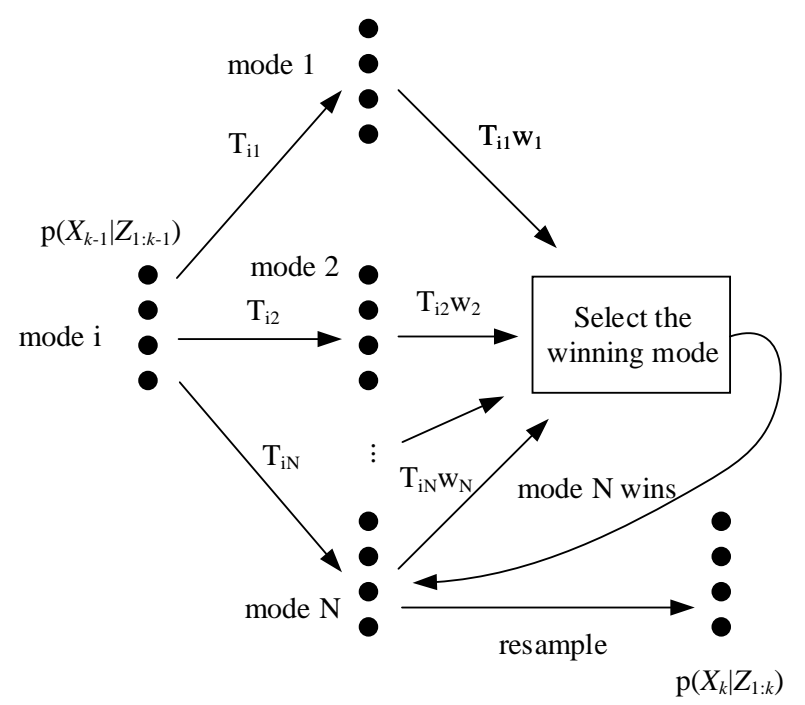

Figure 5. PF for Hybrid System State Estimation

Literature [12] explains the PF method for estimation of dynamical systems in detail. Literature [13] uses a two-tank system to prove the application of the estimation algorithm. The algorithm estimates the mode of the hybrid system and the water levels given the input and output flow. The simulation results demonstrate that it is able to track the state of the system though there is initially some estimation error due to the uncertainty of the initial condition. Literature [14] shows the use of the hybrid estimation algorithm in an aircraft tracking application in air traffic control. The results of the application illustrates that the more accurate the discrete transition model, the better the hybrid estimation accuracy.

It should be noted that PF is computation-intensive in real time applications and may face the problem of particle impoverishment. When it comes to hybrid system estimation, the problem is particularly compounded [15]. The probability of the system to be in mode

$x_{d}$ at time $k$ has a direct relationship with the mode sequences before time $k$ [11]. The number of all possible mode sequences grows exponentially as time progresses. So filter with finite number of particles cannot characterize all possible mode sequences. The mode sequences with low probabilities will be pruned implicitly and passively. It means that the low probability modes cannot be tracked. From a theoretical point of view, the PF will not perform well if the number of particles is small to model a mode sequence. On the other hand, it is a waste to scatter the precious particles among less likely mode sequences. Hence, the better way is to concentrate on the mode sequences with high probabilities.

\section{FDD of Particle Filter}

One of the main difficulties in FDD of the nonlinear stochastic system described by (1) is the presence of unknown and unmeasured state variables $x$ [16]. It has been discussed above that $\mathrm{PF}$ is an excellent tool for state estimation. If the states $x$ of the system are known, the FDD will be an easy task by taking advantage of PF. Now we give the 
definitions of fault detection and diagnosis, then the algorithms for FDD will be listed systematically to give readers a comprehensive understanding of the PF method for FDD of hybrid systems.

Definition 3. Fault detection is to evaluate the $t$ step-ahead prediction distribution:

$$
p\left(X_{k+t} \mid D_{k}\right)=p\left(<x_{c(k+t)}, x_{d(k+t)}>\mid D_{k}\right)
$$

It can be performed using the following equation:

$$
\begin{aligned}
p\left(X_{k+t} \mid D_{k}\right) & =\int p\left(X_{k+t} \mid D_{k}\right) d X_{k+t} \\
& =\int p\left(X_{k} \mid D_{k}\right)\left[\prod_{s=k+t}^{k+t} p\left(X_{s} \mid X_{s-1}\right) d X_{k: k+t-1}\right]
\end{aligned}
$$

Replacing $p\left(X_{k} \mid D_{k}\right)$ in (6) by its approximation obtained by (3), it obtains

$$
p\left(X_{k+t} \mid D_{k}\right)=\sum_{i=1}^{N} w_{k}^{i} \int p\left(X_{k+1} \mid X_{k}^{(i)}\right) \prod_{s=k+2}^{t+k} p\left(X_{s} \mid X_{s-1}\right) d X_{k+1: k+t-1}
$$

where $d X_{k: k+t-1}=d X_{k} \bullet d X_{k+1} \bullet \cdots \bullet d X_{k+t-1}$.

Definition 4. Fault diagnosis is to calculate the posterior distribution $p\left(X_{k} \mid D_{k}\right)=p\left(<x_{c(k)}, x_{d(k)}>\mid D_{k}\right)$. Actually, it has been given in (5). Now we rewrite it here.

$$
\begin{aligned}
p\left(X_{k} \mid D_{k}\right) & =\alpha p\left(D_{k} \mid X_{k}, D_{k-1}\right) p\left(X_{k} \mid D_{k-1}\right) \\
& =\alpha p\left(Z_{k} \mid X_{k}\right) p\left(X_{k} \mid D_{k-1}\right) \\
& =\alpha p\left(Z_{k} \mid X_{k}\right) \sum_{x_{d(k-1)}} \int p\left(X_{k} \mid x_{d(k-1)}, x_{c(k-1)}, D_{k-1}\right) p\left(x_{d(k-1)}, x_{c(k-1)} \mid D_{k-1}\right) d x_{c(k-1)} \\
& =\alpha p\left(Z_{k} \mid X_{k}\right) \sum_{x_{d(k-1)}} \int p\left(X_{k} \mid X_{k-1}\right) p\left(X_{k-1} \mid D_{k-1}\right) d x_{c(k-1)} .
\end{aligned}
$$

Similar $\alpha$ is the normalizing constant.

Now, all the preliminary knowledge has been introduced and it is time to make it clear as to how PF can be used in FDD. Two algorithms will be given next and it is also a summary of the above.

Algorithm 1. Hybrid states estimation algorithm

Let $\left\{X_{k-1}^{(i)}=\left(x_{d(k-1)}^{(i)}, x_{c(k-1)}^{(i)}\right), w_{k-1}^{(i)}, i=1,2, \cdots, N\right\}$ denotes the set of samples at time $k-1 . N$ is the number of the samples. $w_{k-1}^{(i)}$ is the $i$-th sample weight. The hybrid states estimation can be realized as follows:

1) Initialization: Get equal sample weights set $\left\{x_{d(0)}^{(i)}, x_{c(0)}^{(i)}, \frac{1}{N}, i=1,2, \cdots, N\right\}_{i=1}^{N}$ from $p\left(x_{d(0)}\right)$ and $p\left(x_{c(0)}\right)$ at time $k=0$ and set $k=1$.

2) Sequential importance sampling prediction: Calculate $\left\{\tilde{X}_{k \mid k-1}^{(i)}\right\}_{i=1}^{N}$ according to $p\left(X_{k} \mid X_{k-1}^{(i)}\right)$. Here the transition probability function $T_{x_{d(k-1)} x_{d(k)}^{j}}$ is used. Compare the measurements with the output prediction of each particle, and then calculate the importance weight of each sample $w_{k}^{(i)}=p\left(Z_{k} \mid \tilde{X}_{k \mid k-1}^{(i)}\right)$. Next get the normalization $\left\{\tilde{X}_{k \mid k-1}^{(i)}, \tilde{w}_{k \mid k-1}^{(i)}\right\}_{i=1}^{N}$.

3) Resampling: Resample $\mathrm{N}$ equal weighted particles $\left\{X_{k}^{(i)}, \frac{1}{N}\right\}_{i=1}^{N}$ from 
$\left\{\tilde{X}_{k \mid k-1}^{(i)}, \tilde{w}_{k \mid k-1}^{(i)}\right\}_{i=1}^{N}$ according to the size of weight $\tilde{w}_{k \mid k-1}^{(i)}$. In the process, the small weighted particles are discarded.

4) Calculating the transition probability matrix: Compute the autonomous transition probability matrix at next time instant $T\left(x_{d(k+1)} \mid Z_{k}, U_{k}, x_{c(k)}\right)$ using the sample set $\left\{X_{k}^{(i)}, \frac{1}{N}\right\}_{i=1}^{N}$.

5) Hybrid estimation: There is a hybrid estimation at each step. The modes and the continuous states of the system choose the most probability particle to approximate, i.e.

$$
\begin{array}{r}
\hat{x}_{d(k)}=\arg \max _{l} \sum_{i \in \hat{Q}_{l}} w_{k}^{(i)}, \hat{Q}_{l}=\left\{i \mid x_{d(k)}^{(i)}=l\right\} . \\
\hat{x}_{c(k)}=\sum_{i \in \hat{Q}} w_{k}^{(i)} x_{d(k)}^{(i)} / \sum_{i \in \hat{Q}} w_{k}^{(i)}, \hat{Q}=\left\{i \mid x_{d(k)}^{(i)}=\hat{x}_{l}\right\} .
\end{array}
$$

Then set $k=k+1$ and go to step 2).

\section{Algorithm 2. Detection algorithm}

1) Call algorithm 1 to estimate the hybrid states of the system.

2) Compare the output estimation with the real measurement. If the deviation is no more than the threshold or the estimation times are over a set value, then go back step 1) or jump to step 3).

3) Get the deviation between the estimated modes and the observation. If it is bigger than the threshold, a fault can be considered to have occurred, then jump to step 4). If the deviation does not exceed the threshold, we consider that there is no fault in the system now and jump to step 1 ).

4) Give the alarm if a fault happens.

We have finished the discussion on FDD for hybrid systems using PF so far. The methodology talked above gives a framework of FDD for hybrid systems using classical PF or basic PF. Now many efficient variants on the PF algorithm for non-linear hybrid systems have been proposed such as Gaussian sum filter (GSF), Rao-Blackwellised particle filter (RBPF), risk sensitive particle filter (RSPF), variable resolution particle filter (VRPF), look-ahead particle filter (LAPF) and so on. All these methods have their advantages and applicable places. But they are based on the same principle, namely PF. For example, RSPF uses a positive risk function $r(\cdot)$ to generate more particles for highrisk faulted state space. Fault sates will get high $r(\cdot)$ values and so the particles will be more proportionally distributed. The normal operational mode will have a smaller $r(\cdot)$ value and fewer particles will correspondingly be distributed. The data processing ways still continue to use the basic PF algorithm. Refer to the pertinent literatures for detailed information.

\section{Application Examples}

During the past two decades, the PF has always dominated recursive nonlinear state estimation, and has attracted much attention as well as having been widely applied in many fields. The fault diagnosis field is one of the hotspots of PF and much fruitful research has been achieved. In this section, some cases are given to illustrate the application in complex system of FDD using PF. Of course, the methods used in these cases make some modification in PF more or less according to the systems they applied. The application cases quoted in this / are just a brief introduction and please refer to the associated references for technical details. 
One of the most emblematic applications is in robot fault diagnosis. Ref [17] designs a particle filter $(\mathrm{PF})$ based robust navigation with fault diagnosis for an underwater robot. It uses a switching-mode hidden Markov chain models (HMM) to describe the navigation filter design for underwater robots and their anomalies. The experimental results show that the proposed FD method is robust and can diagnose all faults even simultaneous faults within a single structure. Ref. [18] uses PF in a wheeled mobile robot (WMR). It wants to fulfill fault diagnosis and dead reckoning concurrently. PF gives a computationally feasible method for state estimation of hybrid systems. In its methodology, the discussed questions are modeled as a hybrid system estimation problem. By taking advantage of the PF, the discrete states of faults and the continuous states of dead reckoning can be estimated simultaneously.

Another important application is for standalone machines or for the more complex interrelated machinery in large scale industry. As is known to all, industrial systems are all very anfractuous and the failures of them can be catastrophic. So guaranteeing the systems in good state is a crucial objective. Ref. [19] develops a new PF based on dynamic Gaussian mixture model (DGMM) by adopting PF resampling method to update the mixture model parameters in a dynamic fashion. Its superiority is proved by the experimentation in Tennessee Eastman Chemical process.

In addition to that mentioned above, PF can excel in fault prediction or prognosis. Ref. [20] presents a novel method for machinery condition prognosis based on least squares support vector regression combining with strong tracking PF. The strong tracking PF can improve the performance of the PF in tracking saltatory states, and finally strong tracking $\mathrm{PF}$ has verified its superiority in the prediction performance of least squares support vector regression in predicting saltatory states.

\section{Conclusions and Perspectives}

This paper has presented a survey of theoretical results on FDD for hybrid system using PF and some applications have been given to support the theories. The PF-based approach is applicable to general non-linear systems with Gaussian or non Gaussain disturbance noise. The strength of PF such as non-parametric (representing arbitrary distributions easily), dealing with hybrid state spaces, noisy sensing and motion well and extending to an anytime approach easily in the condition of available particles for computation make it attractive for FDD. However, there are still some problems which restrict the development of PF in FDD and need to be further investigated. The paper will be ended in the unanswered problems and its value will be shown if practitioners can get some inspiration from it.

\section{1). From laboratories to real life}

The PF-based method can achieve FDD for hybrid system. But now the current study remains at the theoretical stage, i.e. simulation in computers and experiments in laboratories. There is no known application applied to actual production. The key factors which restrict the application of $\mathrm{PF}$ for FDD in real life lie in the hardware implementation of PF and the real-time application for FDD. It should be the direction for future critical research.

2). Resolve diagnosis in multiple normal modes and fault modes

PF-based method for FDD diagnose faults according to the change of the systems' states. The systems now days are so complex and their operational states and modes are daedal. The normal operational states switch from one mode to another frequently. Most times the mode changes in the systems don't mean failures. This kind of situation brings new challenges for using PF in FDD. Too many modes needing to be tracked can result in 
a huge calculation and probably generate severe particle impoverishment. How to solve the problem is really a headache.

\section{3). Optimize modeling method}

PF-based method for FDD depends on the model of the system largely. The more precise the model is, the more accurate is the diagnosed result. But the modeling for systems especially for complex hybrid systems is a challenging task. If there is no appropriate models for systems, then even good PF-based FDD method is pointless. To find some good modeling methods which can combine with PF may well be a solution to the problem.

\section{4). Lessen calculative burden}

PF realizes the high accuracy of non-linear system states estimation at the expense of sacrificing real-time and increasing the computation. A future direction for further investigation is to refine the PF algorithm with a view to reducing the calculation time whilst maintaining the same precision within the estimation.

\section{Acknowledgements}

The authors would like to thank the support of Natural Science Foundation of China (NO. 61364010). The authors would also like to acknowledge various scholars and their sources which have contributed to this research.

\section{References}

[1] R. Isermann, "Estimation of physical parameters for dynamic processes with application to an industrial robot", International Journal of Control, vol. 55, no. 6, (1992), pp. 1287-1298.

[2] R. Isermann, and P. Balle, "Trends in the application of model-based fault detection and diagnosis of technical processes", Control engineering practice, vol. 5, no. 5, (1997), pp. 709-719.

[3] P. M. Frank, "Fault diagnosis in dynamic systems using analytical and knowledge-based redundancy: A survey and some new results", Automatica, vol. 26, no. 3, (1990), pp. 459-474.

[4] R. Isermann, "Fault-diagnosis systems", Springer, New York, (2006).

[5] R. J. Patton, R. N. Clark, and P. M. Frank, "Issues of fault diagnosis for dynamic systems", Springer Science and Business Media, Berlin, (2013).

[6] N. J. Gordon, D. J. Salmond, and A. F. Smith, "Novel approach to nonlinear/non-Gaussian Bayesian state estimation", IEE Proceedings F (Radar and Signal Processing). IET Digital Library, vol. 140, no. 2, (1993), pp. 107-113.

[7] M. S. Arulampalam, S. Maskell, N. Gordon, and T. Clapp, "A tutorial on particle filters for online nonlinear/non-Gaussian Bayesian tracking”, Signal Processing, IEEE Transactions on, vol. 50, no. 2, (2002), pp. 174-188.

[8] F. Hutter, and R. Dearden, "The gaussian particle filter for diagnosis of non-linear systems", Proceedings of the 5th IFAC Symposium on Fault Detection, Supervision and Safety of Technical Processes, Washington, DC, USA, (2003) June 9-11.

[9] O. B. Martin, B. C. Williams, and M. D. Ingham, "Diagnosis as approximate belief state enumeration for probabilistic concurrent constraint automata", Proceedings of the national conference on artificial intelligence, vol. 20, no. 1, (2005), pp. 321-326.

[10] N. De Freitas, R. Dearden, F. Hutter, R. Morales-Menendez, J. Mutch, and D. Poole, "Diagnosis by a waiter and a mars explorer", Proceedings of the IEEE, vol. 92, no. 3, (2004), pp. 455-468.

[11] S. Tafazoli, and X. Sun, "Hybrid system state tracking and fault detection using particle filters", Control Systems Technology, IEEE Transactions on, vol. 14, no. 6, (2006), pp. 1078-1087.

[12] A, Smith, A. Doucet, N. De Freitas, and N. Gordon, "Sequential Monte Carlo methods in practice", Springer Science and Business Media, Berlin, (2013).

[13] X. Koutsoukos, J. Kurien, and F. Zhao, "Monitoring and diagnosis of hybrid systems using particle filtering methods", International Symposium on Mathematical Theory of Networks and Systems, South Bend, USA, (2002) August 12-16.

[14] C. E. Seah, and I. Hwang, "Stochastic linear hybrid systems: Modeling, estimation, and application in air traffic control", Control Systems Technology, IEEE Transactions on, vol. 17, no. 3, (2009), pp. 563575.

[15] L. Wang, Y. Liang, X. Wang, and L. Xu, "Gaussian sum filter for state estimation of Markov jump nonlinear system”, 17th International Conference on Information Fusion, Salamanca, Spain , (2014) July 
7-10.

[16] M. E. Orchard, and G. J. Vachtsevanos, "A particle-filtering approach for on-line fault diagnosis and failure prognosis", Transactions of the Institute of Measurement and Control, vol. 3, no. 4, (2009), pp. 221-246.

[17] B. Zhao, R. Skjetne, M. Blanke, and F. Dukan, "Particle Filter for Fault Diagnosis and Robust Navigation of Underwater Robot", Control Systems Technology, IEEE Transactions on, vol. 22, no. 6, (2014), pp. 2399-2407.

[18] Z. Duan, Z. Cai, and H. Min, "Robust Dead Reckoning System for Mobile Robots Based on Particle Filter and Raw Range Scan”, Sensors, vol. 14, no. 9, (2014), pp. 16532-16562.

[19] J. Yu, "A particle filter driven dynamic Gaussian mixture model approach for complex process monitoring and fault diagnosis", Journal of Process Control, vol. 22, no. 4, (2012), pp. 778-788.

[20] C. Li, Z. Wang, S. Bu, H. Jiang, and Z. Liu, "A novel method based on least squares support vector regression combing with strong tracking particle filter for machinery condition prognosis", Proceedings of the Institution of Mechanical Engineers, Part C: Journal of Mechanical Engineering Science, vol. 228, no. 6, (2014), pp. 1048-1062.

\section{Author}

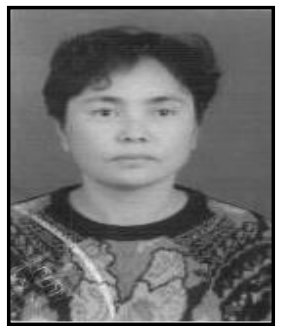

Pazilat Mahmut, received the B.E. degree from Dalian University of Technology, Dalian, China in 1985 and the M.S.E degree from Huazhong University of Science and Technology, Wuhan, China in 2002. She went to Universiteit Twente, Holland as a visiting scholar in 2006 and now she is an associate professor for Xinjiang University, Urumqi, China. Her research interests include fault diagnosis, control theory and applications. 